Running Title: p53 Activation through Stress-induced Signaling

\title{
Signaling to the p53 Tumor Suppressor through Pathways Activated by Genotoxic and Non-genotoxic Stresses
}

Carl W: Anderson ${ }^{1}$ and Ettore Appella ${ }^{2}$

${ }^{1}$ Biology Department, Brookhaven National Laboratory, Upton, NY 11973.

${ }^{2}$ Laboratory of Cell Biology, National Cancer Institute, National Institutes of Health, Bethesda, MD 20892.

\begin{abstract}
.
The p53 tumor suppressor is a tetrameric transcription factor that is posttranslational modified at $\sim 18$ different sites by phosphorylation, acetylation, or sumoylation in response to various cellular stress conditions. Specific posttranslational modifications, or groups of modifications, that result from the activation of different stress-induced signaling pathways are thought to modulate p53 activity to regulate cell fate by inducing cell cycle arrest, apoptosis, or cellular senescence. Here we review the posttranslational modifications to p53 and the pathways that produce them in response to both genotoxic and non-genotoxic stresses.
\end{abstract}

\section{Introduction.}

The product of the human p53 tumor suppressor gene (TP53) is a 393 amino acid polypeptide that functions as a homotetrameric transcription factor. p53 regulates the expression 
of genes that control cell cycle progression, the induction of apoptosis, DNA repair, and other functions involve in cellular responses to stress. Loss of p53 function, either directly through mutation or indirectly through several mechanisms, plays a central role in the development of cancer $[1,2]$. While the 553 protein normally is short-lived and is present at low levels in unstressed mammalian cells, in response to both genotoxic and non-genotoxic stresses it accumulates in the nucleus where it binds to specific DNA sequences [2,3]. Genomic approaches have shown that $\mathrm{p} 53$ induces or inhibits the expression of more than 150 genes including CDKN1A (p21, WAF1, CIP1), GADD45, MDM2, IGFBP3 and BAX [4] that mediate arrest of mammalian cells at one of two major cell cycle checkpoints, in $\mathrm{G}_{1}$ near the border of $\mathrm{S}$ phase, or in $\mathrm{G}_{2}$ before mitosis. 533 modulates DNA repair processes $[5,6]$, and the arrest of cell cycle progression may provide time for the repair of DNA damage. In some circumstances, cell cycle arrest is permanent and indistinguishable from senescence [7]. Alternatively, stress signaling may initiate $\mathrm{p} 53$ dependent apoptosis [2]. The biochemical links between $\mathrm{p} 53, \mathrm{G}_{1}$ arrest, senescence, and apoptosis are cell and stress-type dependent. These observations suggest that specific posttranslational modifications to the 53 protein, at least in part, determine cellular fate. In turn, these modifications reflect the specific pathways that become activated in response to any particular stress condition. In this chapter, we highlight recent studies on the pathways that modulate p53 stability and activity in response to genotoxic and non-genotoxic stresses through covalent posttranslational modifications to 533 including the phosphorylation of serine and threonines and the acetylation of lysines.

\section{II. p53 Protein Structure.}

The $\mathrm{p} 53$ polypeptide can be divided into three functionally distinct regions: an aminoterminal region (1-101, numbering for human p53) that interacts with regulatory proteins and the transcriptional machinery, a central, sequence-specific DNA binding domain (102-292), and a 
carboxyl-terminal tetramerization and regulatory domain (293-393) (Fig. 1). In the $\mathrm{N}$-terminal domain, residues 1-42 are required for transactivation activity and interact with the transcription factors TFIID, TFIIH, several TAFs, the histone acetyltransferases CBP/p300 and possibly PCAF, as well as the MDM2 ubiquitin ligase. Residues 17-28 form an amphipathic helix that interacts directly with a hydrophobic cleft in the N-terminal domain of MDM2 [8], while residues 11-26 are reported to function as a secondary nuclear export signal [9]. Residues 63-97 comprise a proline-rich SH3 domain required for interaction with the Sin3 corepressor [10] and other proteins required for the induction of apoptosis. While residues 1-31 and 80-101 are highly conserved, especially among mammals, residues 32-79 are poorly conserved in sequence even among mammals.

The majority of tumor-derived p53 mutations affect the central domain and block or alter sequence-specific DNA binding either by destabilizing the domain or through changes to residues that directly contact DNA. The three-dimensional structure of the DNA binding domain bound to DNA was determined by X-ray crystallography [11]. The consensus DNA binding site is composed of two $10 \mathrm{bp}$ segments (RRRCWWGYYY) separated by $0-13 \mathrm{bp}$. The carboxyterminal region contains the nuclear localization signal (312-324), a tetramerization domain (323-356) and a basic segment that binds certain DNA structures, including short single strands, four-way junctions, and insertions/deletions in a sequence independent manner [12]. Only tetrameric p53 appears to be active as a transcription factor.

\section{Posttranslational modifications to p53.}

p53 activity is thought to be regulated largely through numerous posttranslational modifications that occur mainly in the $\mathrm{N}$ - and $\mathrm{C}$-terminal regions (Fig. 1). Using monoclonal or affinity-purified polyclonal antibodies produced by several laboratories and companies that recognize specific, modified sites in human or mouse p53, increased phosphorylation at most of 
the 15 known phosphorylation sites has been demonstrated in response to the treatment of cells with DNA damage-inducing agents in Western immunoblot experiments (reviewed in [13]). Seven serines and two threonines in the N-terminal domain of human $\mathrm{p} 53$, specifically Ser6, 9 , $15,20,33,37,46$ and Thr18 and 81 , are phosphorylated in response to exposing cells to ionizing radiation or UV light. Recently, Thr55 was found to be phosphorylated in unstressed cells [14] and dephosphorylated after DNA damage (X. Liu, personal communication). Thus, all Nterminal serines and threonines in the first 89 residues of human p53 may be phosphorylated or dephosphorylated in response to one or more stress conditions. In the C-terminal regulatory domain, Ser315 and Ser392 are phosphorylated, Lys320, 373 and 382 are acetylated, and Lys386 is sumoylated in response to DNA damage. Ser376 and 378 were reported to be constitutively phosphorylated in unstressed cells, while Ser376 was dephosphorylated in response to ionizing radiation [15]. Thr155 and Thr150 or Ser149 in the central site-specific DNA binding domain recently were reported to be phosphorylated by the COP9 signalosome (CSN)-associated kinase [16]; so far, these are the only sites in the central domain that have been reported to be posttranslationally modified.

\section{Regulation of p53 activity.}

The biochemical mechanisms that regulate p53 activity are complex and incompletely understood, but it is widely believed that activation of p53 as a transcription factor involves two stages.

\section{A. p53 stabilization.}

First, in response to stress-activated signaling pathways, p53 is stabilized and accumulates in the nucleus. In unstressed cells, p53 protein is maintained at low steady-state levels and has a short half-live due to rapid, ubiquitin-dependent degradation through the $26 \mathrm{~S}$ proteosome. Ubiquitin is a 76 amino acid polypeptide that is transferred to lysine residues in 
proteins by ubiquitin ligases; multiple ubiquitination targets the protein to the $26 \mathrm{~S}$ proteosome complex where it is degraded. At least three cellular systems that target p53 for ubiquitination have been described. In the $\mathrm{G}_{0}$ phase of the cell cycle, the Jun N-terminal kinase (JNK) binds p53 and targets it for ubiquitination [17]. Activation of JNK in response to DNA damage allows JNK to phosphorylate p53 on Thr81, enhancing its transactivation potential and releasing JNK from $\mathrm{p} 53$. The COP9 signalosome (SSN) recently was shown to bind $\mathrm{p} 53$, phosphorylate it on Thr155 and nearby residues, and promote 553 degradation by targeting it for ubiquitination [16]. Inhibition of the CSN-associated kinase activity or mutation of Thr155 to valine resulted in increased p53 stability and its accumulation. The CSN kinase is thought to be constitutively active; thus, CSN is believe to contribute to the normal turnover of $\mathrm{p} 53$ in cells, but it is not known if modulation of the CSN kinase activity in response to stress contributes to p53 stability.

In dividing cells, the primary system that ubiquitinates p53 is the MDM2 E3 ubiquitin ligase. This activity of MDM2 is vital as shown by the rescue of Mdm2 knock-out mice from embryonic lethality by deletion of $\mathrm{p} 53$. Interestingly, loss of $\mathrm{MdmX}$ expression also results in embryonic lethality that is rescued by deletion of $\mathrm{p} 53$ [18]. MdmX is a structurally related protein that interacts with $\mathrm{Mdm} 2$ and blocks $\mathrm{p} 53$ degradation. A cleft in the $\mathrm{N}$-terminal domain of MDM2 (amino acids 25-109) binds to an amphipathic helix (amino acids 17-29) in the transactivation domain at the $\mathrm{N}$-terminus of $\mathrm{p} 53$ [8], and binding is required for subsequent ubiquitination at multiple C-terminal p53 lysines. p53 is phosphorylated at several $\mathrm{N}$-terminal sites that reside in or near the MDM2 binding site by kinases activated through several stress response pathways; this led to the hypothesis that phosphorylation might stabilize p53 by preventing its interaction with MDM2 [19]. Initially it was proposed that phosphorylation of Ser15 and 37 in response to DNA damage induced a conformational change in p53 that prevented its interaction with MDM2, thus inhibiting p53 ubiquitination [19]. Subsequently, 
phosphorylation of Thr18 and Ser20 were reported to negatively regulated the interaction of p53 with MDM2 [20-23]. Both Thr18 and Ser20 lie within the p53 N-terminal amphipathic helix that directly interacts with the N-terminus of MDM2. Thr18 makes several hydrogen bonds with neighboring residues that stabilize the helix and that would be disrupted by phosphorylation. Consistent with the structural data, phosphorylation of Thr18, but not phosphorylation of Ser15, Ser20, or Ser37, was found to interfere directly with the interaction of an $\mathrm{N}$-terminal p53 peptide with the N-terminal domain of MDM2 [22,24]. Nevertheless, changing Ser15 to alanine was shown to significantly decrease the ability of $\mathrm{p} 53$ to activate transcription and induce apoptosis in both human $[19,25]$ and mouse [26] systems, and changing Ser20 to alanine in human p53 abrogated stabilization in response to DNA damage [20]. Taken together, these results suggest that phosphorylation of $\mathrm{p} 53$ Ser15 and Ser20 may indirectly affect complex formation with Mdm2. One mechanism that would be consistent with the present results is increased competition for binding to the $\mathrm{N}$-terminus of phosphorylated $\mathrm{p} 53$ by other factors. For example, $\mathrm{CBP} / \mathrm{p} 300$ interacts with the $\mathrm{N}$-terminus of $\mathrm{p} 53$, and binding is dramatically enhanced by phosphorylation of Ser15 [27]. Consistent with this finding, changing Ser6, Ser9, Ser15, or Thr18 (but not more distal serines) to alanine reduced acetylation of Lys382 [28]. Surprisingly, however, changing murine Ser23, the equivalent of human Ser20, to alanine had no measurable affect on transactivation, apoptosis, and Mdm2 binding in mouse ES cells, fibroblasts, or thymocytes [29]. Thus, it spite of the high degree of sequence conservation between human and mouse p53 in this region, the two species appear to regulate p53 stabilization and MDM2 binding somewhat differently. Several other proteins have been shown to interact with the Nterminus of p53 (see Fig. 1), but the effect of p53 phosphorylation on their binding has not be examined.

Recent results suggest that other mechanism also play an important role in regulating 
MDM2 activity. First, p14 ${ }^{\mathrm{ARF}}$ can inhibit the activity of MDM2 by sequestering it in the nucleolus or through other mechanisms [30]. Second, MDM2 itself is a target for DNA damage induced posttranslational modifications that may positively or negatively regulate its activity [31]. Third, several studies have shown that the C-terminal regulatory region of p53 is important for its stabilization. The circumstances under which each mechanism operates and their relative importance in regulating p53 stability have not be fully characterized, but the existence of several mechanisms may explain reported differences in results relating to different phosphorylation sites.

A fourth system that targets $\mathrm{p} 53$ for ubiquitin-mediated degradation is the E6 protein of human papillomaviruses. Papillomaviruses are small, DNA tumors viruses, and certain serotypes are strongly associated with cervical cancer. Like other DNA tumor viruses, papillomaviruses inactivate $\mathrm{p} 53$, both to allow cells to enter a state in which the DNA viral genome can replicate and to avoid virus-induced apoptosis. The papillomavirus E6 protein, in conjunction with a $\sim 100$ $\mathrm{kDa}$ cellular protein $\mathrm{E} 6 \mathrm{AP}$, functions as a ubiquitin ligase in a manner similar to MDM2 [32]. As a consequence, papillomavirus transformed human cells (e.g. HeLa) frequently have wildtype p53 genes but are functionally deficient for p53 activity.

\section{B. p53 activation.}

p53 accumulation alone is not sufficient to fully activate p53-dependent transcription (see [33]). Early studies by Hupp et al. indicated that p53 is synthesized in a latent form that is incompetent for sequence-specific DNA binding [34]; they proposed that subsequent to its synthesis p53 was activated to a DNA-binding competent state through posttranslational modifications that were postulated to induce a conformational change in the DNA binding domain of $\mathrm{p} 53$. Indeed, several modifications to the C-terminus, including phosphorylation [34], acetylation $[35,36]$, binding of antibodies, and truncation of the C-terminal 30 amino acids, were 
shown to result in increased $\mathrm{p} 53$ binding to oligonucleotides containing the p53 consensus recognition site. These results suggested that the $\mathrm{C}$-terminal domain negatively regulated sequence-specific binding by the central domain. The C-terminal regulatory domain of p53 contains numerous basic residues, and subsequently it was found to bind single-stranded DNA and RNA, as well as several DNA structures. An alternative model to explain latency was proposed by Anderson et al [37], who showed that the sequence-specific DNA binding of p53 was inhibited by long DNAs but not by short oligonucleotides (Fig. 2, top). These results suggested that strong non-sequence-specific binding by the C-terminal domain of tetrameric p53 prevented sequence-specific binding. Furthermore, relief of inhibition of sequence-specific DNA binding through removal of the C-terminus only occurred in the presence of long DNA molecules. The interference model recently received indirect support from NMR-based structural studies on a dimeric derivative of $\mathrm{p} 53$ and its $\mathrm{C}$-terminal truncated form [38]. No significant differences in the structure of the central DNA binding domain were observed between the two forms, suggesting that increased DNA binding did not result from an allosteric conformational change in $\mathrm{p} 53$ structure. However, the concept of a latent DNA binding form of $\mathrm{p} 53$ recently was challenged by the finding that unmodified p53 binds well to long DNAs containing consensus recognition sites [39]. Indeed, p53 was reported to be constitutively bound to chromatin at some recognitions sites in vivo, including sites in the CDKN1A (p21,WAF1,CIP1), and MDM2 promoters [40]. Furthermore, genotoxic stress caused only a small increase in the amount of p53 bound to chromatin at these sites.

If DNA/chromatin binding is not the rate limiting step for activation of $\mathrm{p} 53$, then what might this step be? Although direct attempts to demonstrate a role for p53 acetylation in transcriptional activation in vivo have been unsuccessful [41], the importance of histone acetyltransferases (HATs) for p53 transcriptional activity was demonstrated by overexpression of 
the histone deacetylases HDAC-1, -2 , or -3 , or hSir2, which deacetylate p53 and inhibit the transcription of p53-target genes [42-46]. These and other recent results suggest an alternative model for $\mathrm{p} 53$ mediated transcriptional activation in which p53 serves to target HATs to the promoters of $\mathrm{p} 53$ activated target genes (Fig. 2, bottom). Likewise, the targeting of HDACs by p53 to p53 repressed genes has been suggested as a mechanism of p53-mediate gene repression [47]. The mechanisms by which HATs (and HDACs) associate with p53 are not completely understood. As noted above, we previously proposed that DNA damage induced $N$-terminal phosphorylations promote the association of p300/CBP with p53 [36]. However, other targeting mechanisms may exist. Recently it was suggested that acetylated residues in the p53 C-terminus may serve to recruit co-activators [48].

A role for p53 in targeting the co-activator p300 to chromatin is consistent with recent in vitro transcription studies by Espinosa and Emerson [39]. They found that a plasmid containing the $C D K N 1 A(p 21)$ promoter, which contains two p53 binding sites located 2.3 and $1.5 \mathrm{~kb}$ proximal of the transcription start site, was not transcribed efficiently when assembled intochromatin. Transcription was strongly enhanced, however, by addition of both p53 and p300 but not by either factor alone. Importantly, unacetylated p 53 bound efficiently to the plasmid DNA and with even higher affinity to the nucleosomal template, but not to a 25 base pair oligonucleotide containing the $5^{\prime} p 21$ promoter consensus site. Transcriptional activation required the $\mathrm{C}$-terminus of $\mathrm{p} 53$ but not acetylation of $\mathrm{C}$-terminal sites. Furthermore, p53 was shown to induce acetylation of nucleosomal histones preferentially in the promoter region of the chromosomal template, consistent with the model depicted in Fig. 2 (bottom). The authors [39] suggested that binding of $\mathrm{p} 53$ to consensus sites in long DNA might be facilitated by the ability of long DNA, but not short oligonucleotides, to form non-B-DNA structures [49] or to bend DNA [50], as suggested previously. Surprisingly, however, several p53 activated promoters 


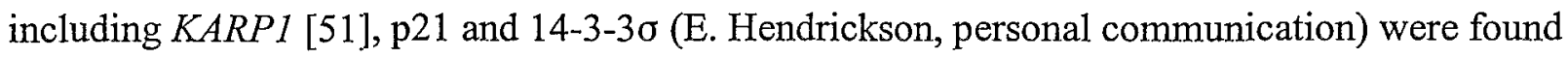
to be in an "open" conformation as judged by the presence of DNAse I hypersensitive sites in these promoters prior to $\mathrm{p} 53$ activation by treatment of cells with DNA damage-inducing agents. DNAse I chromatin hypersensitivity is a hallmark of promoter regions of actively transcribed genes, where as the promoter regions of inactive genes usually are insensitive to DNAse I.

\section{Activation of $\mathrm{p53}$ by genotoxic stresses.}

Mammalian cells appear to have at least two largely independent signaling pathways for activating p53 in response to genotoxic stress; one is activated by the presence of DNA doublestrand breaks, the other in response to bulky lesions such as pyrimidine dimers and base adducts.

\section{A. Ionizing radiation.}

Treatment of cells with ionizing radiation or several radiomimetics (e.g. neocarzinostatin, bleomycin) activates several kinases that phosphorylate p53 at multiple sites (Fig. 3). Although the molecular mechanism(s) by which DNA strand breaks are recognized are still obscure, key among the kinases activated in response to DNA breaks is ATM, a protein kinase member of the phosphatidylinositol-3-kinase kinase family encoded by the gene responsible for the human genetic disorder ataxia telangiectasia (A-T). ATM directly phosphorylates p53 at Ser15 and activates several other protein kinases that phosphorylate the $\mathrm{N}$-terminal transactivation domain including Chk1 and Chk2, which phosphorylate p53 at Ser20 and perhaps other residues (Fig. 1). ATM appears to activate other, unidentified protein kinases that directly phosphorylated Ser9 and Ser46 [28]. Protein kinases that directly phosphorylate Ser6, Ser33, Ser37, and Ser315 appear to be activated in response to DNA double-strand breaks by ATM-independent pathways, although inactivation of possible site-specific phosphatases for these residues in response to DNA strand breaks cannot be ruled out. Induction of phosphorylation through substrate 
modification also is possible. Casein kinase 1 (CK1) phosphorylates sites two residues distal to a previously phosphorylated serine or threonine. In vitro p53 previously phosphorylated at Ser6 and Ser15 is phosphorylated by CK1 at Ser9 or Thr18, respectively [22,52]. Thus, CK1, or a similar activity, may provide a mechanism for phosphorylation site signal amplification, at least in some cells. Several kinases, including CAK (Ser33), CDK (Ser315), PKA, and PKC (Ser376, Ser378), that are capable of phosphorylating p53 in vitro have been identified (Fig. 1), but if and how these might be activated in response to DNA strand breaks is unknown (reviewed in [13]).

DNA-PK, another PI-3K family member that phosphorylates p53 at both Ser15 and Ser37 in vitro, is directly activated by DNA strand breaks through targeting to DNA ends by its $\mathrm{Ku}$ subunit, but its role in vivo remains uncertain. A recent study reported that $\mathrm{p} 53$ is found in a complex that contains nucleotide analogue-modified DNA and activated DNA-PK [53]. Treatment of cells with the analogue induced kinase activity, phosphorylation of $\mathrm{p} 53$ on Ser15 resulting in its stabilization and activation, and triggered apoptosis. Consistent with this report, Woo et al. recently reported that DNA-PK is required for the IR-mediated apoptosis of mouse embryonic fibroblasts [54]. However, DNA-PK is not required for p53-mediated cell cycle arrest in $G_{1}$ or the induction of $p 53$ activated transcription in response to ionizing radiation [55].

\section{B. UV light.}

A second DNA damage response pathway is activated in response to bulky lesions, such as the pyrimidine dimers caused by UV-C (Fig. 3). As for IR, the molecular mechanism(s) responsible for sensing UV damaged DNA are not known, although the pathway is though to involve sensing a block to transcriptional elongation [56] and activation of a third PI-3 kinase family member, ATR (A-T and Rad3-related) [57]. Recently, it was reported that ATR exhibits

preferential binding to UV-damaged DNA; thus, under some circumstance, it may act directly as 
a sensor of UV damage [58]. In vitro ATR phosphorylates p53 on Ser15 and Ser37. UV-C also induces p53 phosphorylation on Ser6, 9, 20, 33, 46, 315, 392, and on Thr18 and 81 (Fig. 3); thus exposure of cells to UV light must activate or induces a number of other kinases that phosphorylate p53. Among these are p38 MAPK, which targets Ser33 and Ser46 [59], HIPK2, which also targets Ser46 [60,61], JNK, which phosphorylates Thr81 [17], and a complex containing casein kinase 2 (CK2) and the transcription elongation factor FACT that phosphorylates Ser392 [62]. In response to UV light, each of these sites is phosphorylated in cells lacking ATM ([28] and Saito et al., unpublished). The dependence of phosphorylation at other sites on ATR has not been carefully investigated since ATR-deficient cells are not viable. Phosphorylation at Ser46 correlates with the induction of apoptosis and the transcriptional induction of $A I P I$ (p53 regulated apoptosis inducing protein 1) by UV-C [63]. Substitution of Ser46 with alanine inhibited p53-mediated apoptosis and the induction of AIP1. Evidence has been presented that the majority of $\mathrm{p} 53$ protein can be phosphorylated at Ser 315 by cdc $2 / \mathrm{Cdk} 2$ in response to UV-induced DNA damage and that the cyclin-dependent kinases play a role in stimulating p53 function [64]. In contrast to UV-C, UV-A triggers activation of p53 through activation of the ATM kinase activity, apparently through the production of reactive oxygen species [65].

\section{Carboxy-terminal DNA damage-induced p53 modifications.}

As noted above, two C-terminal PKC sites, Ser376 and 378, were reported to be constitutively phosphorylated in the absence of DNA damage [15]. Thr55 also may be constitutively phosphorylated and then dephosphorylated in response to DNA damage. Treatment of MFC7 cells with IR led to the ATM-dependent dephosphorylation of Ser376 and to the association of p53 with a 14-3-3 protein, which increased its in vitro affinity for sequencespecific DNA. A confounding observation is the fact that p53 from unstressed cells is recognized 
by the monoclonal antibody PAb421, which recognizes an epitope including Lys372 to Lys382, but recognition is inhibited by phosphorylation of Ser376 or Ser378 (and acetylation of Lys382). Recently $\mathrm{H7}$, an inhibitor of PKC, was reported to prevent p53 ubiquitination and to stimulate p53 accumulation in cells without inducing its activation, suggesting that the constitutive phosphorylation of p53 at Ser376 and/or Ser378 contributes to its degradation [66]. The function of UV-mediated phosphorylation of Ser392 is not clear, as previously Ser392 was shown not to be required for the suppression of cell growth or the activation of transcription [67]. In vitro, phosphorylation of Ser392 stimulated formation of p53 tetramers, while phosphorylation of Ser315 reversed this effect [68]; however, these effects are seen at low p53 concentrations and may have been masked in cells overexpressing exogenous $\mathrm{p} 53$.

The acetylation of several C-terminal p53 lysines is stimulated by several forms of stress including the genotoxic damage caused by both UV and IR $[36,69]$. These residues including Lys320, which is acetylated by PCAF in vitro, and Lys373 and Lys382, which can be acetylated by $\mathrm{p} 300 / \mathrm{CBP}$. Prior to the discovery of DNA damage-mediated p53 acetylation, CBP/p300 and PCAF were shown to be co-activators of p53-mediated transcription. As noted above, C-terminal acetylation is promoted by the phosphorylation of N-terminal sites, especially Ser15, but acetylation at Lys382 also was enhanced by phosphorylation at nearby residues, including Ser6, Ser9, and Thr18 [28]. However, in A549 cells acetylation was not induced equivalently by IR and UV. Acetylation, especially of Lys320, was much more strongly induced by UV than IR. Potential roles for $\mathrm{p} 53$ acetylation include contributions to $\mathrm{p} 53$ stability through interference with C-terminal ubiquitination and the modulation of $\mathrm{p} 53$ 's interactions with other proteins and DNA. Possible roles for p53 in targeting HATs and HDACs to chromatin are discussed above and illustrated in Fig. 2. 


\section{Other genotoxic agents.}

Because of their convenience, ionizing radiation and UV light commonly are used in the laboratory to produce two different forms to genotoxic damage, i.e. DNA double-strand breaks and pyrimidine dimers. Many other environmental, physiological, and therapeutic agents cause genotoxic damage that activate p53 through one or more signaling pathways. These include anticancer drugs such as adriamycin, topoisomerase inhibitors such as camptothecin, etoposide, and quercetin, DNA synthesis and transcription inhibitors including aphidicolin, actinomycin D and 5,6-dichloro-1-beta-D-ribofuranosylbenzimidazole (DRB), DNA cross-linking agents such as cisplatinin and mitomycin $\mathrm{C}$, and environmental chemicals including arsnite, cadmium, and chromate. Each of the above agents have been shown to induces p53 accumulation and its phosphorylation on Ser15 except DRB, which interferes with phosphorylation of the CTD domain of RNA polymerase II rather than with elongation [70]. For most of these agents, however, modifications to $\mathrm{p} 53$ at other sites have not been investigated.

\section{Activation of p53 by non-genotoxic stresses.}

p53 activation occurs in response to several physiological processes that are not associated with frank DNA damage, including hypoxia, nucleotide deprivation, microtubule inhibitors, oncogene activation and senescence potentiated by telomere erosion. Some of these processes may be mimicked by pharmacological agents which may or may not induce identical responses.

\section{A. Hypoxia.}

Tumor hypoxia occurs in most solid tumors from abnormal vasculature development; hypoxia also is an important pathophysiological feature of ischemic disorders. Hypoxia and several hypoxia mimetics have been shown to induce p53 accumulation as a result of the downregulation of MDM2 [71] with concomitant phosphorylation of Ser15, but not acetylation of 
Lys382 [72] (Fig. 3). However, in contrast to IR, hypoxia treatment failed to induce the transcription of downstream effector mRNAs including GADD45, Bax, and p21 [72]. Hypoxia does not induce detectable DNA damage, and, in contrast to DNA damage-inducing agents, primarily caused an association of $\mathrm{p} 53$ with $\mathrm{mSin} 3 \mathrm{~A}$ rather than $\mathrm{p} 300$. Consistent with this finding, p53-mediated transrepression was induced. In hypoxia treated human papillomavirus HPV-16 transformed cells, p53 was resistant to E6-mediated degradation, and its association with E6AP was reduced [71]. Interestingly, a recent study has shown that inhibition of ATR kinase activity reduced the hypoxia-induced phosphorylation of p53 protein on Ser15 as well as p53 protein accumulation [73]. These data suggest that hypoxia could select for the loss of ATRdependent checkpoint controls, thus promoting cell transformation.

\section{B. Ribonucleotide depletion.}

Studies by Linke et al. [74] showed that p53 is activated in normal human fibroblasts by the N-phosphoacetyl-L-aspartate-induced (PALA) depletion of ribonucleotides in the absence of detectable DNA damage. In contrast to the $\mathrm{G}_{1}$ arrest induced by DNA damage, that induced by PALA was readily reversible. PALA treatment induced a pattern of gene expression that was distinct from that induced by IR. Some of these, such as $M D G I$, a mammary-derived growth inhibitor gene, were induced independent of $\mathrm{p} 53$ while for others, such as TSG6, a tumor necrosis factor stimulated gene, induction was $\mathrm{p} 53$ dependent [75]. The modification status of p53 after PALA treatment has not been address.

\section{Microtubule disruption.}

Activation of p53 also occurs in response to factors such as colcemid, nocodazole, and taxol that deregulate cell adhesion or microtubule architecture and dynamics. Taxol (Paclitaxel), which inhibits microtubule depolymerization, is one of the newer chemotherapy drugs 
commonly is used to treat ovarian, breast, and head and neck cancers. After nocodazole treatment, which depolymerizes microtubules, quiescent human fibroblasts accumulated transcriptionally active p53 and arrested in $\mathrm{G}_{1}$ with a $4 \mathrm{~N}$ DNA content [76]. Activation of p53 after colcemid treatment was accompanied by a moderate increase in phosphorylation at Ser 15 and correlated with activation of Erk1/2 MAP kinases and the development of focal adhesions rather than disruption of the microtubule system [77]. Curiously, murine fibroblasts did not undergo the same response. Taxol and vincristine, but not nocodazole, were found to induced multi-site phosphorylation of p53 in several tumor-derived human cell lines, including HCT-116 and RKO cells, and the pattern of p53 phosphorylation was distinct from that observed after DNA damage [78]. Nevertheless, both nocodozole and taxol increased phosphorylation at Ser15 (Fig. 3). Interestingly, microtubule inhibitor-induced p53 stabilization and Ser15 phosphorylation did not occur in ATM-deficient fibroblasts nor in normal human dermal fibroblasts. Studies with ectopically expressed p53 phosphorylation site mutants indicated that several p53 aminoterminal residues, including Ser15 and Thr18, were required for the taxol-mediated phosphorylation of p53 [78]. In contrast, Damia et al. [79] reported that taxol induced p53 phosphorylation at Ser20 but not at Ser15 in HCT-116 cells. Phosphorylation at Ser20 was accompanied by increased Chk2 activity and was not inhibited in A-T cells lines nor by wortmannin treatment. Thus, the signaling pathways that impinge on p53 after hypoxia, ribonucleotide depletion or microtubule disruption, while still not well defined, appear distinct from those induced by genotoxic stresses.

\section{Oncogene activation.}

Oncogenes, such as Ras, c-Myc or E1a, when activated or overexpressed, stabilize and activate p53, and depending on the cell type, induce senescence (Ras) or apoptosis (c-Myc or E1a) through hyperproliferative signaling pathways that activate ARF, the product of the 
alternative reading frame of the cell-cycle regulatory gene INK4a/CDKN2a [30]. ARF, in turn, inhibits $\mathrm{p} 53$ degradation by MDM2. Based on the observation that $\mathrm{p} 53$ Ser15 was not phosphorylated in response to adenovirus E1A expression, it was concluded that oncogenic activation of p53 occurs in absence of DNA damage [80]. However, in normal fibroblasts brief c_Myc overexpression induced DNA damage prior to $\mathrm{S}$ phase that correlated with the induction of reactive oxygen species [81], raising the question of whether oncogenes activate p53 through DNA damage and whether the ability of oncogenes to promote either apoptosis or senescence correlates with different p53 posttranslational modifications. Ferbeyre et al. [82] reported that expression of oncogenic Ras induced phosphorylation of Ser15 in IMR90 cells. In contrast, Bulavin et al [83] found p53 was phosphorylated at Ser33 and Ser46 but not at other N- or Cterminal sites, nor was it acetylated at Lys382 (Fig. 3). Interestingly, a similar induction of permanent cell cycle arrest resembling cellular senescence was produced in murine fibroblasts engineered to express the MAP kinase Mek1 [84]. The induction of senescence by Ras required wildtype p53 and ARF, but p53 was not required to maintain the senescent state. These data indicate that other signals may influence the outcome of p53 activation, likely by changing its association with various coactivators; however, whether this leads to the expression of different p53 target genes remains to be determined.

\section{E. Replicative senescence.}

Replicative senescence in human fibroblasts correlates with activation of p53-dependent transcription and was shown to be associated with increased phosphorylation at Ser15, Thr18, and probably Ser376, and decreased phosphorylation at Ser392 [85] (Fig. 3). It was inferred from the finding that no change occurred in staining with the DO-1 monoclonal antibody, the epitope for which includes Ser20, that phosphorylation on Ser20 was not induced. These results, in conjunction with findings showing that changes in $\mathrm{p} 53$ phosphorylation are abrogated in cells 
which have been immortalized by overexpression of telomerase, indicate that the above modifications may be the product of telomere erosion. Shorten or disrupted telomere structures may signal to p53 via pathways partially shared with DNA damage responses.

\section{Conclusions.}

Multiple, distinct signal transduction pathways clearly activate and modulate p53dependent transcription in response to both genotoxic and non-genotoxic stresses. Although key protein kinases that are likely to phosphorylate p53 in response to DNA damage have been identified, the identities of kinases that phosphorylate several important sites are still unknown. Furthermore, several sites may be phosphorylated by more than one protein kinase. This complexity is augmented further by the facts that signaling pathway activation may be cell-type and cell-cycle dependent and that many signaling initiation events activate more than one pathway.

A fundamental question that remains unanswered is what mechanism(s) contribute to the ability of different cells to interpret $\mathrm{p} 53$ activation in different ways. The activation of $\mathrm{p} 53$ by hypoxia or oncogenes clearly induces different effects than the response to genotoxic stresses. Therefore, the pattern of posttranslational modifications may determine the selection of the subsets of target genes regulated in response to p53 activation, but a precise understanding of the mechanisms is not yet in hand. It is clear that the p53 protein forms complexes with many other cellular components and with particular nuclear structures. This characteristic may influence the degree of its activation and contribute to the heterogeneity of p53-dependent responses observed within a specific tissue. The analysis of the modification patterns in different mouse tissues of knock-in mutants should give insights as to the role played by individual phosphorylations and acetylations sites in eliciting a molecular signaling outcome. While there is still much to learn, 
substantial progress in understanding the cause and effects of $\mathrm{p} 53$ response responses is being made.

\section{References}

1. Hollstein, M., Sidransky, D., Vogelstein, B., and Harris, C. C. (1991). p53 mutations in human cancers. Science, 253, 49-53.

2. Bálint, É. E., and Vousden, K. H. (2001). Activation and activities of the p53 tumour suppressor protein. Br. J. Cancer, 85, 1813-1823.

3. Wahl, G. M., and Carr, A. M. (2001). The evolution of diverse biological responses to DNA damage: insights from yeast and p53. Nat. Cell Biol., 3, E277-286.

4. Zhao, R., Gish, K., Murphy, M., Yin, Y., Notterman, D., Hoffman, W. H., Tom, E., Mack, D. H., and Levine, A. J. (2000). Analysis of p53-regulated gene expression patterns using oligonucleotide arrays. Genes Dev., 14, 981-993.

5. Hanawalt, P. C. (2001). Controlling the efficiency of excision repair. Mutat. Res., 485, 313.

6. Smith, M. L., and Seo, Y. R. (2002). p53 regulation of DNA excision repair pathways. Mutagenesis, 17, 149-156.

7. Campisi, J. (2001). Cellular senescence as a tumor-suppressor mechanism. Trends Cell. Biol., 11, S27-31.

8. Kussie, P. H., Gorina, S., Marechal, V., Elenbaas, B., Moreau, J., Levine, A. J., and Pavletich, N. P. (1996). Structure of the MDM2 oncoprotein bound to the p53 tumor suppressor transactivation domain. Science, 274, 948-953.

9. Zhang, Y., and Xiong, Y. (2001). A p53 amino-terminal nuclear export signal inhibited by DNA damage-induced phosphorylation. Science, 292, 1910-1915. 
10: Zilfou, J. T., Hoffman, W. H., Sank, M., George, D. L., and Murphy, M. (2001). The corepressor $\mathrm{mSin} 3 \mathrm{a}$ interacts with the proline-rich domain of $\mathrm{p} 53$ and protects $\mathrm{p} 53$ from proteasome-mediated degradation. Mol. Cell. Biol., 21, 3974-3985.

11. Cho, Y., Gorina, S., Jeffrey, P. D., and Pavletich, N. P. (1994). Crystal structure of a p53 tumor suppressor-DNA complex: understanding tumorigenic mutations. Science, 265, 346-355.

12. Wolkowicz, R., and Rotter, V. (1997). The DNA binding regulatory domain of p53: see the C. Pathol. Biol. (Paris), 45, 785-796.

13. Appella, E., and Anderson, C. W. (2001). Post-translational modifications and activation of p53 by genotoxic stresses. Eur. J. Biochem., 268, 2764-2772.

14. Gatti, A., Li, H.-H., Traugh, J. A., and Liu, X. (2000). Phosphorylation of human p53 on Thr-55. Biochemistry, 39, 9837-9842.

15. Waterman, M. J. F., Stavridi, E. S., Waterman, J. L. F., and Halazonetis, T.D. (1998). ATM-dependent activation of $\mathrm{p} 53$ involves dephosphorylation and association with 14-33 proteins. Nat. Genet., 19, 175-178.

16. Bech-Otschir, D., Kraft, R., Huang, X., Henklein, P., Kapelari, B., Pollmann, C., and Dubiel, W. (2001). COP9 signalosome-specific phosphorylation targets p53 to degradation by the ubiquitin system. EMBO J., 20, 1630-1639.

17. Buschmann, T., Potapova, O., Bar-Shira, A., Ivanov, V. N., Fuchs, S. Y., Henderson, S., Fried, V. A., Minamoto, T., Alarcon-Vargas, D., Pincus, M. R., Gaarde, W. A., Holbrook, N. J., Shiloh, Y., and Ronai, Z. (2001). Jun $\mathrm{NH}_{2}$-terminal kinase phosphorylation of $\mathrm{p} 53$ on Thr- 81 is important for $\mathrm{p} 53$ stabilization and transcriptional activities in response to stress. Mol. Cell. Biol., 21, 2743-2754.

18. Parant, J., Chavez-Reyes, A., Little, N. A., Yan, W., Reinke, V., Jochemsen, A. G., and Lozano, G. (2001). Rescue of embryonic lethality in Mdm4-null mice by loss of Trp53 
suggests a nonoverlapping pathway with MDM2 to regulate p53. Nat. Genet., 29, 92-95.

19. Shieh, S.-Y., Ikeda, M., Taya, Y., and Prives, C. (1997). DNA damage-induced phosphorylation of p53 alleviates inhibition by MDM2. Cell, 91, 325-334.

20. Chehab, N. H., Malikzay, A., Stavridi, E. S., and Halazonetis, T. D. (1999). Phosphorylation of Ser-20 mediates stabilization of human p53 in response to DNA damage. Proc. Natl. Acad. Sci. US A, 96, 13777-13782.

21. Craig, A. L., Burch, L., Vojtesek, B., Mikutowska, J., Thompson, A., and Hupp, T. R. (1999). Novel phosphorylation sites of human tumour suppressor protein $\mathrm{p} 53$ at $\operatorname{Ser}^{20}$ and $\mathrm{Thr}^{18}$ that disrupt the binding of $\mathrm{mdm} 2$ (mouse double minute 2) protein are modified in human cancers. Biochem. J., 342, 133-141.

22. Sakaguchi, K., Saito, S., Higashimoto, Y., Roy, S., Anderson, C. W., and Appella, E. (2000). Damage-mediated phosphorylation of human p53 threonine 18 through a cascade mediated by a casein 1-like kinase. Effect on Mdm2 binding. J. Biol. Chem., 275, 92789283.

23. Unger, T., Juven-Gershon, T., Moallem, E., Berger, M., Vogt Sionov, R., Lozano, G., Oren, M., and Haupt, Y. (1999). Critical role for Ser20 of human p53 in the negative regulation of $\mathrm{p} 53$ by $\mathrm{Mdm} 2$. EMBO J., 18, 1805-1814.

24. Lai, Z., Auger, K. R., Manubay, C. M., and Copeland, R. A. (2000). Thermodynamics of p53 binding to hdm2(1-126): effects of phosphorylation and p53 peptide length. Arch. Biochem. Biophys., 381, 278-284.

25. Fiscella, M., Ullrich, S.J., Zambrano, N., Shields, M. T., Lin, D., Lees-Miller, S. P., Anderson, C. W., Mercer, W. E., and Appella, E. (1993). The carboxy-terminal serine 392 phosphorylation site of human p53 is not required for wild-type activities. Oncogene, 8, 1519-1528.

26. Chao, C., Saito, S., Anderson, C. W., Appella, E., and Xu, Y. (2000). Phosphorylation of 
murine 553 at Ser-18 regulates the p53 responses to DNA damage. Proc. Natl. Acad. Sci. $U S A, 97,11936-11941$.

27. Lambert, P. F., Kashanchi, F., Radonovich, M. F., Shiekhattar, R., and Brady, J. N. (1998). Phosphorylation of p53 serine 15 increases interaction with CBP. J. Biol. Chem., 273, 33048-33053.

28. Saito, S., Goodarzi, A.A., Higashimoto, Y., Noda, Y., Lees-Miller, S. P., Appella, E., and Anderson, C. W. (2002). ATM mediates phosphorylation at multiple p53 sites, including $\mathrm{Ser}^{46}$, in response to ionizing radiation. J. Biol. Chem., 277, 12491-12494.

29. Wu, Z., Earle, J., Saito, S., Anderson, C. W., Appella, E., and Xu, Y. (2002). Mutation of mouse p53 Ser23 and the response to DNA damage. Mol. Cell. Biol., 22, 2441-2449.

30. Sherr, C.J. (2001). The INK4a/ARF network in tumour suppression. Nat. Rev. Mol. Cell Biol., 2, 731-737.

31. Michael, D., and Oren, M. (2002). The p53 and Mdm2 families in cancer. Curr. Opin. Genet. Dev., 12, 53-59.

32. Talis, A.L., Huibregtse, J. M., and Howley, P. M. (1998)'. J. Biol. Chem., 273, 6439-6445.

33. Agarwal, M. L., Taylor, W. R., Chernov, M. V., Chernova, O. B., and Stark, G. R. (1998). The role of E6AP in the regulation of $\mathrm{p} 53$ protein levels in human papillomavirus (HPV)-positive and HPV-negative cells. J. Biol. Chem., 273, 1-4.

34. Hupp, T. R., and Lane, D. P. (1994). Allosteric activation of latent p53 tetramers. Curr. Biol., 4, 865-875.

35. Gu, W., and Roeder, R. G. (1997). Activation of p53 sequence-specific DNA binding by acetylation of the p53 C-terminal domain. Cell, 90, 595-606.

36. Sakaguchi, K., Herrera, J. E., Saito, S., Miki, T., Bustin, M., Vassilev, A., Anderson, C. W., and Appella, E. (1998). DNA damage activates p53 through a phosphorylationacetylation cascade. Genes Dev., 12, 2831-2841. 
37. Anderson, M. E., Woelker, B., Reed, M., Wang, P., and Tegtmeyer, P. (1997). Reciprocal interference between the sequence-specific core and nonspecific C-terminal DNA binding domains of p53: implications for regulation. Mol. Cell. Biol., 17, 6255-6264.

38. Ayed, A., Mulder, F. A. A., Yi, G.-S., Lu, Y., Kay, L. E., and Arrowsmith, C. H. (2001). Latent and active p53 are identical in conformation. Nat. Struct. Biol., 8, 756-760.

39. Espinosa, J. M., and Emerson, B. M. (2001). Transcriptional regulation by p53 through intrinsic DNA/chromatin binding and site-directed cofactor recruitment. Mol. Cell, 8, 5769.

40. Kaeser, M. D., and Iggo, R. D. (2002). Chromatin immunoprecipitation analysis fails to support the latency model for regulation of 53 DNA binding activity in vivo. Proc. Natl. Acad. Sci. US A, 99, 95-100.

41. Nakamura, S., Roth, J. A., and Mukhopadhyay, T. (2000). Multiple lysine mutations in the C-terminal domain of 553 interfere with MDM2-dependent protein degradation and ubiquitination. Mol. Cell. Biol., 20, 9391-9398.

42. Juan, L.-J., Shia, W.-J., Chen, M.-H., Yang, W.-M., Seto, E., Lin, Y.-S., and Wu, C.-W. (2000). Histone deacetylases specifically down-regulate p53-dependent gene activation. J. Biol. Chem., 275, 20436-20443.

43. Luo, J., Su, F., Chen, D., Shiloh, A., and Gu, W. (2000). Deacetylation of p53 modulates its effect on cell growth and apoptosis. Nature, 408, 377-381.

44. Luo, J., Nikolaev, A.Y., Imai, S., Chen, D., Su, F., Shiloh, A., Guarente, L., and Gu, W. (2001). Negative control of p53 by Sir2 $\alpha$ promotes cell survival under stress. Cell, 107, $137-148$.

45. Vaziri, H., Dessain, S. K., Eaton, E. N., Imai, S. I., Frye, R. A., Pandita, T. K., Guarente,

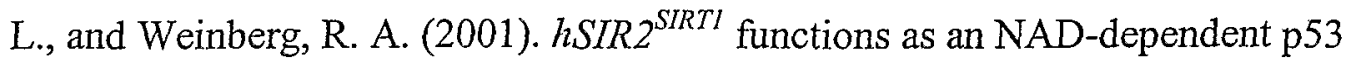
deacetylase. Cell, 107, 149-159. 
46. Langley, E., Pearson, M., Faretta, M., Bauer, U.-M., Frye, R. A., Minucci, S., Pelicci, P. G., and Kouzarides, T. (2002). Human SIR2 deacetylates p53 and antagonizes PML/p53induced cellular senescence. EMBO J., 21, 2383-2396.

47. Murphy, M., Ahn, J., Walker, K. K., Hoffman, W. H., Evans, R. M., Levine, A. J., and George, D. L. (1999). Transcriptional repression by wild-type p53 utilizes histone deacetylases, mediated by interaction with mSin3a. Genes Dev., 13, 2490-2501.

48. Barlev, N. A., Liu, L., Chehab, N. H., Mansfield, K., Harris, K. G., Halazonetis, T. D., and Berger, S. L. (2001). Acetylation of p53 activates transcription through recruitment of coactivators/histone acetyltransferases. Mol. Cell, 8, 1243-1254.

49. Kim, E., Rohaly, G., Heinrichs, S., Gimnopoulos, D., Meißner, H., and Deppert, W. (1999). Influence of promoter DNA topology on sequence-specific DNA binding and transactivation by tumor suppressor p53. Oncogene, 18, 7310-7318.

50. Nagaich, A. K., Zhurkin, V. B., Durell, S. R., Jernigan, R. L., Appella, E., and Harrington, R. E. (1999). p53-induced DNA bending and twisting: p53 tetramer binds on the outer side of a DNA loop and increases DNA twisting. Proc. Natl. Acad. Sci. US A, 96, $1875-1880$.

51. Braastad, C. D., Leguia, M., and Hendrickson, E. A. (2002). Ku86 autoantigen related protein- 1 transcription initiates from a $\mathrm{CpG}$ island and is induced by $\mathrm{p} 53$ through a nearby p53 response element. Nucleic Acids Res., 30, 1713-1724.

52. Higashimoto, Y., Saito, S., Tong, X.-H., Hong, A., Sakaguchi, K., Appella, E., and Anderson, C. W. (2000). Human p53 is phosphorylated on serines 6 and 9 in response to DNA damage-inducing agents. J. Biol. Chem., 275, 23199-23203.

53. Achanta, G., Pelicano, H., Feng, L., Plunkett, W., and Huang, P. (2001). Interaction of p53 and DNA-PK in response to nucleoside analogues: potential role as a sensor complex for DNA damage. Cancer Res., 61, 8723-8729. 
54. Woo, R. A., Jack, M. T., Xu, Y., Burma, S., Chen, D. J., and Lee, P. W. K. (2002). DNA damage-induced apoptosis requires the DNA-dependent protein kinase, and is mediated by the latent population of p53. EMBO J., 21, 3000-3008.

55. Jimenez, G. S., Bryntesson, F., Torres-Arzayus, M. I., Priestley, A., Beeche, M., Saito, S., Sakaguchi, K., Appella, E., Jeggo, P. A., Taccioli, G. E., Wahl, G. M., and Hubank, M. (1999). DNA-dependent protein kinase is not required for the p53-dependent response to DNA damage. Nature, $400,81-83$.

56. Ljungman, M., Zhang, F., Chen, F., Rainbow, A. J., and McKay, B. C. (1999). Inhibition of RNA polymerase II as a trigger for the p53 response. Oncogene, 18, 583-592.

57. Abraham, R. T. (2001). Cell cycle checkpoint signaling through the ATM and ATR kinases. Genes Dev., 15, 2177-2196.

58. Ünsal-Kaçmaz, K., Makhov, A. M., Griffith, J. D., and Sancar, A. (2002). Preferential binding of ATR protein to UV-damaged DNA. Proc. Natl. Acad. Sci. US A, 99, 66736678.

59. Bulavin, D. V., Saito, S., Hollander, M. C., Sakaguchi, K., Anderson, C. W., Appella, E., and Fornace Jr, A. J. (1999). Phosphorylation of human p53 by p38 kinase coordinates Nterminal phosphorylation and apoptosis in response to UV radiation. EMBO J., 18, 68456854.

60. D'Orazi, G., Cecchinelli, B., Bruno, T., Manni, I., Higashimoto, Y., Saito, S., Gostissa, M., Coen, S., Marchetti, A., Del Sal, G., Piaggio, G., Fanciulli, M., Appella, E., and Soddu, S. (2002). Homeodomain-interacting protein kinase-2 phosphorylates p53 at Ser 46 and mediates apoptosis. Nat. Cell Biol., 4, 11-19.

61. Hofmann, T. G., Möller, A., Sirma, H., Zentgraf, H., Taya, Y., Dröge, W., Will, H., and Schmitz, M. L. (2002). Regulation of p53 activity by its interaction with homeodomaininteracting protein kinase-2. Nat. Cell Biol., 4, 1-10. 
62. Keller, D. M., Zeng, X., Wang, Y., Zhang, Q. H., Kapoor, M., Shu, H., Goodman, R., Lozano, G., Zhao, Y., and Lu, H. (2001). A DNA damage-induced p53 serine 392 kinase complex contains CK2, hSpt16, and SSRP1. Mol. Cell, 7, 283-292.

63. Oda, K., Arakawa, H., Tanaka, T., Matsuda, K., Tanikawa, C., Mori, T., Nishimori, H., Tamai, K., Tokino, T., Nakamura, Y., and Taya, Y. (2000).p53AIP1, a potential mediator of p53-dependent apoptosis, and its regulation by Ser-46-phosphorylated p53. Cell, 102, 849-862.

64. Blaydes, J. P., Luciani, M. G., Pospisilova, S., Ball, H. M.-L., Vojtesek, B., and Hupp, T. R. (2001). Stoichiometric phosphorylation of human p53 at $\mathrm{Ser}^{315}$ stimulates p53dependent transcription. J. Biol. Chem., 14, 4699-4708.

65. Zhang, Y., Ma, W.-Y., Kaji, A., Bode, A. M., and Dong, Z. (2002). Requirement of ATM in UVA-induced signaling and apoptosis. J. Biol. Chem., 277, 3124-3131.

66. Chernov, M. V., Bean, L. J. H., Lerner, N., and Stark, G. R. (2001). Regulation of ubiquitination and degradation of p53 in unstressed cells through C-terminal phosphorylation. J. Biol. Chem., 276, 31819-31824.

67. Fiscella, M., Zambrano, N., Ullrich, S., Unger, T., Lin, D., Cho, B., Mercer, W., Anderson, C., and Appella, E. (1994). Mutation of the serine 15 phosphorylation site of human p53 reduces the ability of p53 to inhibit cell cycle progression. Oncogene, 9 , $3249-3257$.

68. Sakaguchi, K., Sakamoto, H., Xie, D., Erickson, J. W., Lewis, M. S., Anderson, C. W., and Appella, E. (1997). Effect of phosphorylation on tetramerization of the tumor suppressor protein p53. J. Protein Chem., 16, 553-556.

69. Ito, A., Lai, C.-H., Zhao, X., Saito, S., Hamilton, M. H., Appella, E., and Yao, T.-P. (2001). p300/CBP-mediated p53 acetylation is commonly induced by p53-activating agents and inhibited by MDM2. EMBO J., 20, 1331-1340. 
70. Ljungman, M., O'Hagan, H. M., and Paulsen, M. T. (2001). Induction of ser15 and lys 382 modifications of p53 by blockage of transcription elongation. Oncogene, 20, 5964-5971.

71. Alarcón, R., Koumenis, C., Geyer, R. K., Maki, C. G., and Giaccia, A. J. (1999). Hypoxia induces p53 accumulation through MDM2 down-regulation and inhibition of E6mediated degradation. Cancer Res., 59, 6046-6051.

72. Koumenis, C., Alarcon, R., Hammond, E., Sutphin, P., Hoffman, W., Murphy, M., Derr, J., Taya, Y., Lowe, S. W., Kastan, M., and Giaccia, A. (2001). Regulation of p53 by hypoxia: dissociation of transcriptional repression and apoptosis from p53-dependent transactivation. Mol. Cell. Biol., 21, 1297-1310.

73. Hammond, E. M., Denko, N. C., Dorie, M. J., Abraham, R. T., and Giaccia, A. J. (2002). Hypoxia links ATR and p53 through replication arrest. Mol. Cell. Biol., 22, 1834-1843.

74. Linke, S. P., Clarkin, K. C., Di Leonardo, A., Tsou, A., and Wahl, G. M. (1996). A reversible, $\mathrm{p} 53$-dependent $\mathrm{G}_{0} / \mathrm{G}_{1}$ cell cycle arrest induced by ribonucleotide depletion in the absence of detectable DNA damage. Genes Dev., 10, 934-947.

75. Seidita, G., Polizzi, D., Costanzo, G., Costa, S., and Di Leonardo, A. (2000). Differential gene expression in $\mathrm{p} 53$-mediated $\mathrm{G}_{1}$ arrest of human fibroblasts after $\gamma$-irradiation or $N$ phosphoacetyl-L-aspartate treatment. Carcinogenesis, 21, 2203-2210.

76. Khan, S. H., and Wahl, G. M. (1998). p53 and pRb prevent rereplication in response to microtubule inhibitors by mediating a reversible $\mathrm{G}_{1}$ arrest. Cancer Res., 58, 396-401.

77. Sablina, A. A., Chumakov, P. M., Levine, A. J., and Kopnin, B. P. (2001). p53 activation in response to microtubule disruption is mediated by integrin-Erk signaling. Oncogene, 20, 899-909.

78. Stewart, Z. A., Tang, L. J., and Pietenpol, J. A. (2001). Increased p53 phosphorylation after microtubule disruption is mediated in a microtubule inhibitor- and cell-specific 
manner. Oncogene, 20, 113-124.

79. Damia, G., Filiberti, L., Vikhanskaya, F., Carrassa, L., Taya, Y., D'incalci, M., and Broggini, M. (2001). Cisplatinum and taxol induce different patterns of p53 phosphorylation. Neoplasia, 3, 10-16.

80. de Stanchina, E., McCurrach, M. E., Zindy, F., Shieh, S. Y., Ferbeyre, G., Samuelson, A. V., Prives, C., Roussel, M. F., Sherr, C. J., and Lowe, S. W. (1998). E1A signaling to p53 involves the p19(ARF) tumor suppressor. Genes Dev., 12, 2434-2442.

81. Vafa, O., Wade, M., Kern, S., Beeche, M., Pandita, T. K., Hampton, G. M., and Wahl, G. M. (2002). c-myc can induce DNA damage, increase reactive oxygen species, and mitigate p53 function. A mechanism for oncogene-induced genetic instability. Mol. Cell, 9, 1031-1044.

82. Ferbeyre, G., de Stanchina, E., Querido, E., Baptiste, N., Prives, C., and Lowe, S. W. (2000). PML is induced by oncogenic ras and promotes premature senescence. Genes Dev. 14, 2015-2027.

83. Bulavin, D. V., Demidov, O. N., Saito, S., Kauraniemi, P., Phillips, C., Amundson, S. A., Ambrosino, C., Sauter, G., Nebreda, A. R., Anderson, C. W., Kallioniemi, A., Fornace, Jr., A. J., and Appella, E. (2002). Amplification of PPMID in human tumors abrogates p53 tumor-suppressor activity. Nat. Genet., 31, 210-215.

84. Ferbeyre, G., de Stanchina, E., Lin, A. W., Querido, E., McCurrach, M. E., Hannon, G. J., and Lowe, S. W. (2002). Oncogenic ras and p53 cooperate to induce cellular senescence. Mol. Cell. Biol., 22, 3497-3508.

85. Webley, K., Bond, J. A., Jones, C. J., Blaydes, J. P., Craig, A., Hupp, T., and WynfordThomas, D. (2000). Posttranslational modifications of p53 in replicative senescence overlapping but distinct from those induced by DNA damage. Mol. Cell. Biol., 20, 28032808 . 
86. Stommel, J. M., Marchenko, N. D., Jimenez, G. S., Moll, U. M., Hope, T. J., and Wahl, G. M. (1999). A leucine-rich nuclear export signal in the p53 tetramerization domain: regulation of subcellular localization and p53 activity by NES masking. EMBO J., 18, $1660-1672$.

\section{Figure Legends}

Figure 1. Protein domains, posttranslational modification sites and proteins that interact with human p53. The 393 amino acid human p53 polypeptide is represented schematically (box) with the five most highly conserved regions marked (I-V); postulated function regions and domains also are indicated. Residues $\sim 1-42$ comprise the transactivation domain; residues $\sim 63-97$ constitute a Src homology 3-like (SH3) domain that overlaps a poorly conserved proline and alanine rich segment (33-80); residues $\sim 102-292$ contain the central, sequence-specific, DNA binding core region; residues $\sim 300-323$ contain the primary nuclear localization signal (NLS); residues 324-356 comprise the tetramerization domain (TET) which contains a nuclear export signal [86]; residues 363-393 (REG) negatively regulate DNA binding by the central core to consensus recognition sites in oligonucleotides and interact in a sequence-independent manner with single- and double-stranded nucleic acids. Interactions regions for selected proteins are indicated below the polypeptide, and posttranslational modification sites $(\mathrm{P}$, phosphorylation; Ac, acetylation) are indicated above the peptide together with enzymes that can accomplish the modifications in vitro. Lys386 may be modified by conjugation with SUMO1, a ubiquitin-like peptide. References are found in the text and recent reviews.

Figure 2. Models for the activation of $\mathrm{p} 53$ as a transcription factor. A (upper scheme). Regulation of DNA binding by the C-terminal domain. Unmodified p53 exists as a latent 
tetramer incapable of sequence-specific DNA binding. Upon modification of C-terminal residues by phosphorylation and/or acetylation, latent $\mathrm{p} 53$ is converted into an active, sequence-specific, DNA binding protein that then recruits elements of the transcription apparatus (e.g. TAFs). The conversion of latent p53 into active p53 might occur through an allosteric transition in the DNAbinding domain [34], or as a consequence of the inhibition of non-specific DNA binding to the C-terminal regulatory domain [37]. Recent NMR studies [38] argue against activation through an allosteric transition. B (lower scheme). Recent studies suggest that p53 may be constitutively bound at some p53 consensus sites; thus, latency of DNA binding may be an artifact of the in vitro conditions employed [40]. Other studies have shown that both histone acetyltransferase (HAT) coactivators, such as $\mathrm{CBP} / \mathrm{p} 300$, and histone deacetylase (HDAC) corepressors are recruited to $\mathrm{p} 53$ through stress-induced posttranslational modifications to the p53 $\mathrm{N}$-terminus. Although $\mathrm{CBP} / \mathrm{p} 300$ and PCAF acetylate lysines at the C-terminus of $\mathrm{p} 53$, mutation of these sites failed to demonstrate a strong effect on p53-mediated transcription [38]. Instead, recruitment of HATs and HDACs to promoter sites may alter chromatin structure in the vicinity of p53 promoters, suggesting that activation of p53-mediated transcription may result from the action of these coactivators and repressors on the modification state and structure of chromatin in the vicinity of $\mathrm{p} 53$ promoters [39].

Figure 3. Posttranslational modifications to $\mathrm{p} 53$ in response to genotoxic and non-genotoxic stress. The bar at the bottom represents the human 393 amino acid p53 polypeptide; functional regions are indicated. Reported posttranslational modification sites are indicated above the bar; $\mathrm{S}$, serine; $\mathrm{T}$, threonine; $\mathrm{K}$, lysine. Filled circles (phosphorylation) or squares (acetylation) indicate modification in response to the indicated stress (left); open symbols indicate no change in modification in response to stress. No symbol indicates the site has not been examined. Selected references are given at the right. A "?" indicates conflicting literature reports; a down 
arrow indicates treatment induced a decrease in site modification. Thr55 [14] and Ser376 and 378 [15] were reported to be constitutively phosphorylated; Thr55 and Ser376 are dephosphorylated in response to DNA damage. Ser6, 9, 33, 315, and 392 may be constitutively phosphorylated at low levels in some cell lines (Saito et al., unpublished).

\section{Acknowledgments}

We thank Sharlyn J. Mazur for constructive suggestions. We apologize to those whose publications could not be cited due to space limitations. C.W.A. was supported in part by a CRADA funded by the Laboratory Technology Research Program in the Office of Science of the U.S. Department of Energy.

Corresponding Author: Ettore Appella, Laboratory of Cell Biology, National Cancer Institute, National Institutes of Health, Bethesda, MD 20892; Phone 301-402-4177; Fax: 301-496-7220; email: ea3v@nih.gov 


\section{Human p53 Tumor Suppressor Protein}

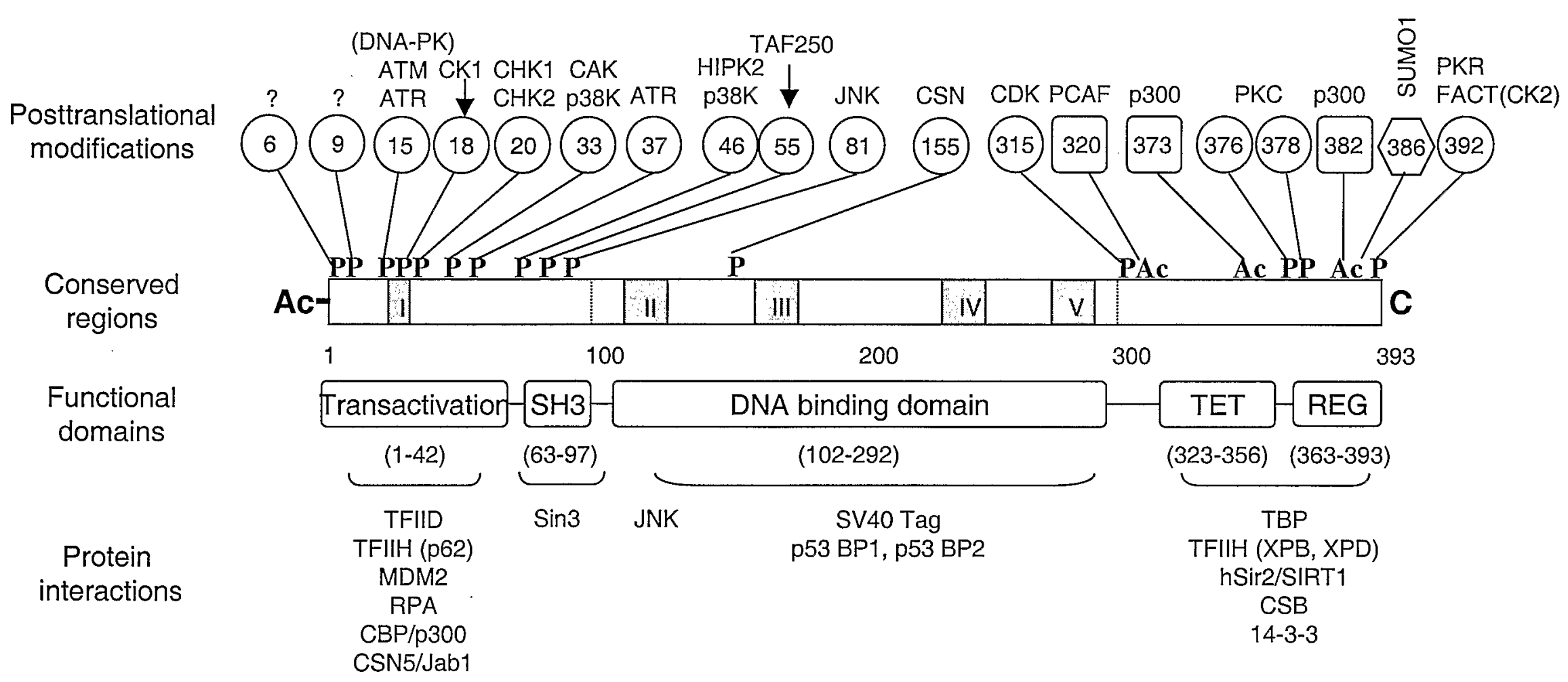

Anderson and Appella Figure 1 


\section{Models for the Activation of p53}

Model A: Latent DNA Binding
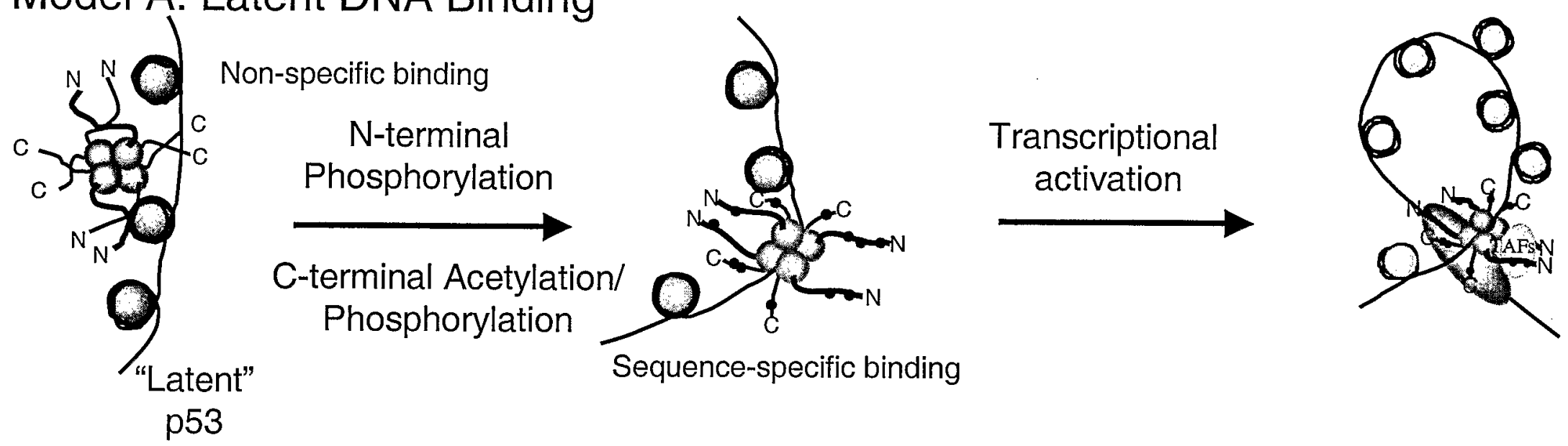

Model B: HAT/HDAC Recruitment and Chromatin Remodeling

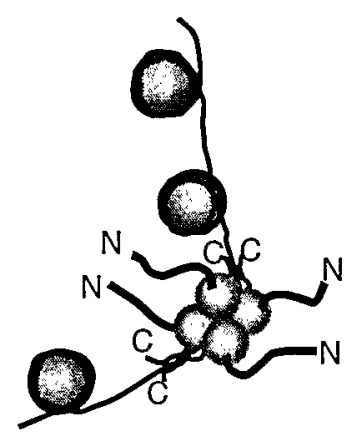

"Latent" p53

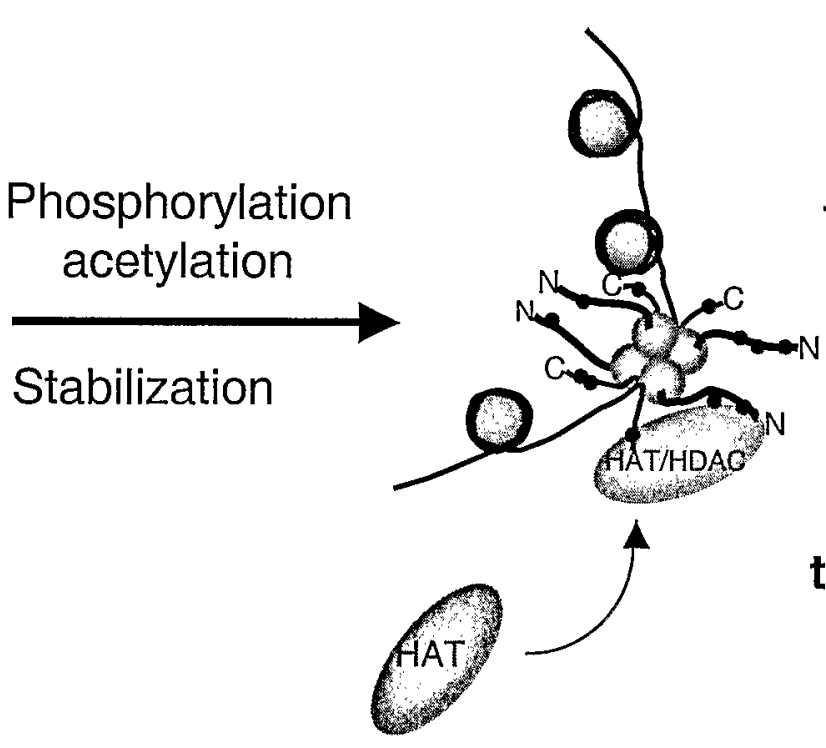

Co-activators (p300/CBP)

transcriptional activation

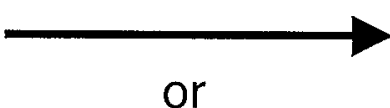

Co-repressors

Sin3, HDAC

transcriptional repression

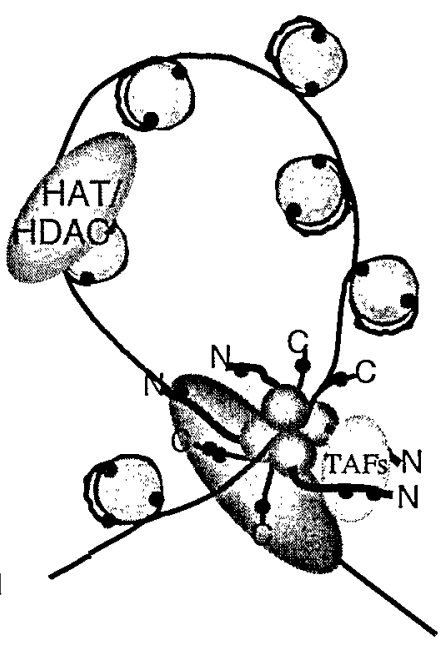

Anderson and Appella Figure 2 


\section{Posttranslational modifications to p53 in response to genotoxic and non-genotoxic stress}

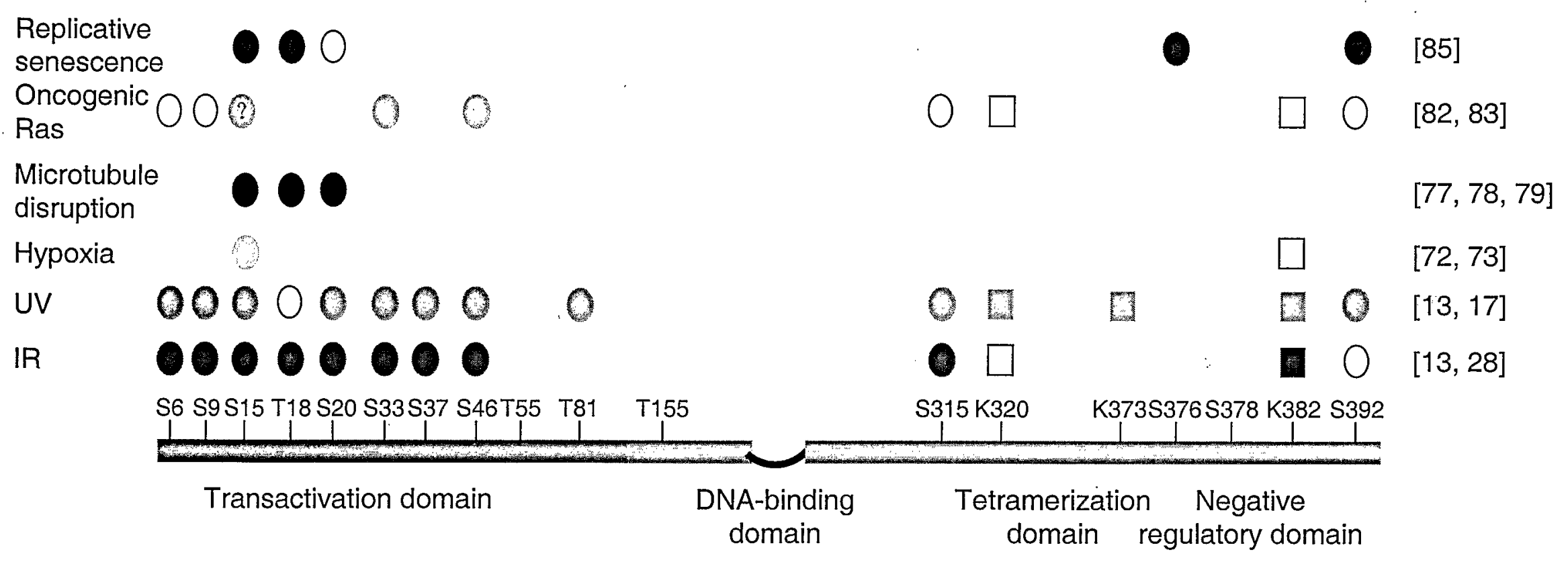

Anderson and Appella Figure 3 